\title{
ESTIMACIÓN DE LA DEMANDA DE AGUA EN CENTROS EDUCATIVOS: CASO DE ESTUDIO FACULTAD DE CIENCIAS AMBIENTALES DE LA UNIVERSIDAD TECNOLÓGICA DE PEREIRA, COLOMBIA
}

\author{
Deibys Manco-Silva \\ Jhoniers Guerrero-Erazo (D) 2 \\ Tito Morales-Pinzón (D) 3
}

Recibido el 12 de marzo de 2015, aprobado el 16 de mayo de 2016 y actualizado el 15 de diciembre de 2016

\section{DOI: 10.17151/luaz.2017.44.9}

\section{RESUMEN}

La gestión del agua en centros educativos debe partir de la identificación y cuantificación de la demanda de agua según los diferentes usos existentes en la institución. En este artículo se presenta la caracterización de la demanda de agua en la Facultad de Ciencias Ambientales de la Universidad Tecnológica de Pereira, identificando los usuarios y sus hábitos de consumo, con el propósito de generar procesos de comprensión y herramientas de gestión que permitan iniciar programas en torno al uso eficiente del agua. En primer lugar, se identificaron los elementos que conforman el sistema de abastecimiento de agua potable en el edificio y se clasificaron los usuarios del agua. Bajo técnicas de observación, diálogo con los usuarios y la instalación de medidores volumétricos de media pulgada R-160, se midieron los consumos de cada uno de los usuarios. A partir de esta información, se determinó la demanda del centro educativo, así como la demanda de agua para cada uno de los usos. Finalmente, se calcularon indicadores de demanda y se propone un modelo matemático para el cálculo del consumo de agua en centros educativos.

\section{PALABRAS CLAVE}

Centros educativos, demanda de agua, gestión del recurso hídrico, usuarios.

\section{WATER DEMAND ESTIMATION IN EDUCATION CENTERS: A CASE STUDY IN ENVIRONMENTAL SCIENCE FACULTY OF TECHNOLOGICAL UNIVERSITY OF PEREIRA, COLOMBIA}

\begin{abstract}
Water management in educational centers involves identifying and quantifying water demand for different uses given in those institutions. This paper presents the characterization of water demand in the Environmental Sciences Faculty, Technological University of Pereira, identifying users and their habits, in order to generate understanding processes and management tools that allow starting programs related to water efficiency. First, the project identified the elements of the water supply system in the building and the different water uses were classified. Under observation techniques, dialogue with users and installing a half inch volumetric meters R-160, were measured water consumption of each user. From this information it was determined the consumption and demand for each use in the faculty. Finally, demand indicators were calculated and it was proposed a mathematical model for the calculation of water consumption in an educative center.
\end{abstract}




\section{KEY WORDS}

Educative center, water demand, water management resource, users.

\section{INTRODUCCIÓN}

El abastecimiento de agua potable es condición indispensable para el desarrollo de la vida humana. Los usuarios del agua en zonas urbanas se clasifican en: residenciales, comerciales, industriales e institucionales. Dentro de esta clasificación de uso, las instituciones o centros educativos, por su tamaño y demanda de agua son considerados como altos consumidores (Cheng y Hong, 2004). Para ilustrar, Bonet et al (2002), señalan que los consumos de agua en centros universitarios son similares a los consumos de ciudades de tamaño medio. Así mismo, con el fin de promover procesos de conservación y uso eficiente en centros educativos, Cheng y Hong (2004) identificaron los altos consumos de agua en escuelas primarias como uno de los principales problemas a resolver. Con lo anterior, se espera obtener beneficios ambientales como la disminución de la presión en la demanda del recurso hídrico y la diminución en las descargas generadas a las fuentes hídricas receptoras (Manco, Guerrero y Ocampo, 2012).

La gestión del agua en estas instituciones implica un control eficiente y eficaz, tanto en los sistemas de distribución como en los usuarios (Jorgensen, Graymore y O'toole, 2009). En este orden de ideas, con el fin de iniciar un proceso de gestión de la demanda de agua en la Universidad Tecnológica de Pereira (UTP) y tomando como caso de estudio la Facultad de Ciencias Ambientales (FCA), se caracterizaron los usuarios y los hábitos de consumo de agua, con el fin de generar procesos de comprensión y herramientas e indicadores de gestión.

\section{MATERIALES Y MÉTODOS}

\section{Área de Estudio}

El campus de la UTP se encuentra localizado en la ciudad de Pereira, departamento de Risaralda, Colombia; la temperatura ambiente promedio es de $22^{\circ} \mathrm{C}$ y la altura sobre el nivel del mar es de 1.411 metros. Su población universitaria para el año 2011 fue de 14.956 estudiantes, 1.482 docentes, 317 administrativos, comprende un área de $502.122 \mathrm{~m}^{2}$ y cuenta con nueve facultades, entre las que está la FCA, la cual presentaba una población de 1.031 personas, entre estudiantes (997), docentes y administrativos (34). Comprende un área de $5.185 \mathrm{~m}^{2}$, lo que representa el $1 \%$ del área total del campus universitario (figura 1). 
Figura 1. Localización Facultad Ciencias Ambientales

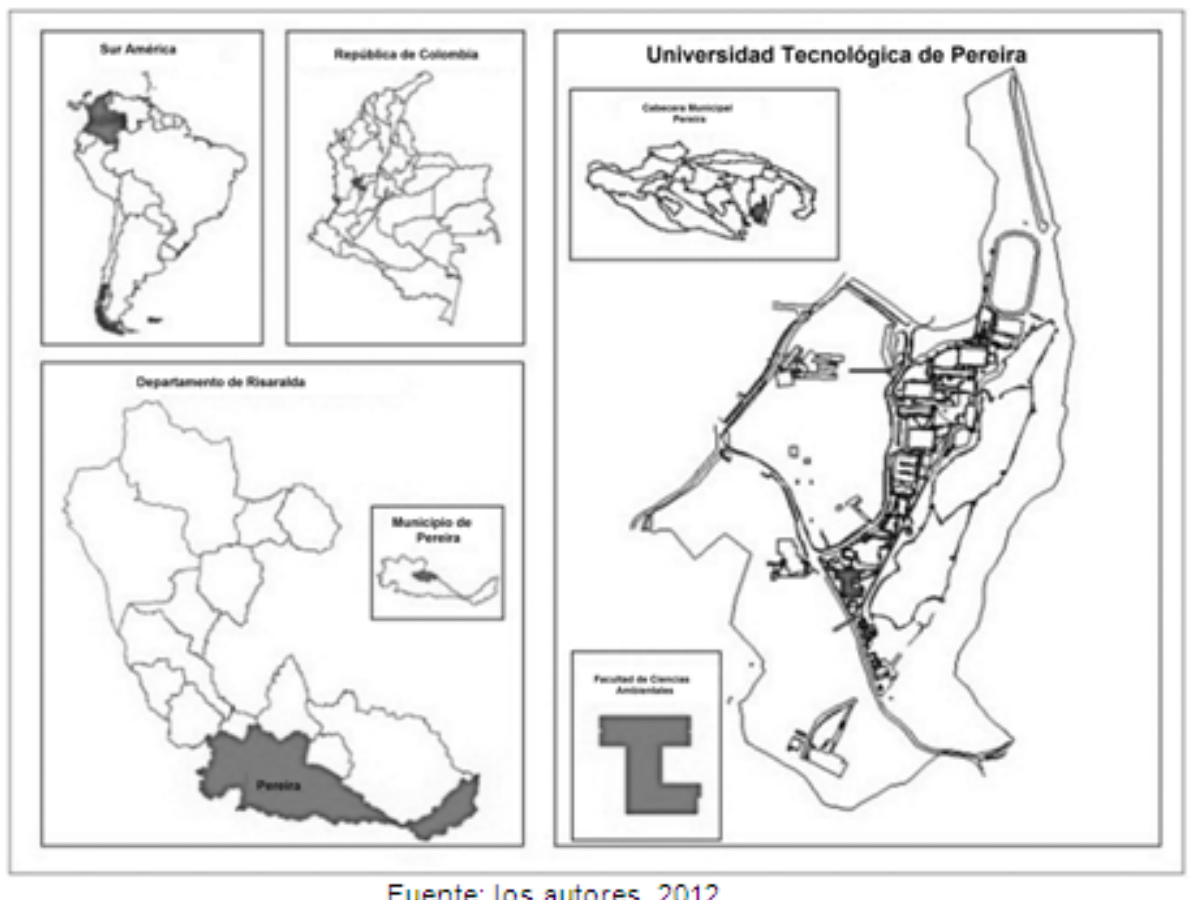

\section{Metodología}

Con el propósito de comprender las dinámicas que se dan en torno a la demanda de agua en la FCA, se procedió a identificar aquellos elementos que conforman el sistema de distribución de agua potable como son tuberías, estructuras y dispositivos (instalaciones hidrosanitarias) que sirven para proveer de agua a la comunidad universitaria.

Mediante técnicas de observación directa y registro (visitas de campo, diálogo con estudiantes, docentes, personal de limpieza y mantenimiento), se realizó el inventario de las instalaciones hidráulicas ${ }^{1}$ para estimar el tipo de instalación (grifos, urinarios, inodoros y duchas), al igual que su estado, es decir, si presentan o no fugas importantes o goteos, y si cuentan con algún tipo de equipamiento tecnológico, reductor de caudal o regulador de volumen (aireador, tecla de interrupción o válvula de doble descarga).

Así mismo, se identificó y clasificó a los usuarios del agua con el fin de determinar en qué y cómo se usa el agua en la FCA. De esta manera se especificaron los siguientes usos: aseo personal (estudiantes, docentes y administrativos); preparación de alimentos (cafetería docentes y administrativos, dispensador de agua); riego de jardín; laboratorios y limpieza.

Con el fin de cuantificar el consumo de agua para cada uso, se instalaron en cada unidad hidrosanitaria medidores volumétricos $R$-160 de media pulgada $(1 / 2$ ") con módulo radio IZAR CP $434 \mathrm{MHz}$ (emisor de pulso), que envía los registros de consumo de agua a la unidad concentradora de datos Gprs Receiver, estos quedan disponibles en el Servidor FTP y vía internet pueden ser consultados y descargados (figura 2).

Se realizó un muestreo desde el día 23 de agosto hasta el 09 de septiembre de 2011, durante este segundo semestre académico se presentó un funcionamiento normal del 
centro educativo. En cada unidad de servicio o aprovisionamiento de agua, se registraron los consumos de agua hora a hora. La información recolectada fue tabulada y analizada en el software Izar Task ${ }^{\circledR}$ y Microsoft Exce/®, que permitieron procesar los datos y construir las curvas de perfil del consumo para cada uso.

A partir de los datos recolectados y haciendo uso del modelo de simulación Plugrisost, desarrollado por Morales-Pinzón et al. (2012a), se realizaron simulaciones sobre diferentes configuraciones de demanda de agua en centros educativos. Estos resultados fueron analizados usando el software PASW Statistics 18 - SPSS mediante la técnica estadística de análisis de regresión lineal con el propósito de ajustar un modelo general que fuera aplicable a diferentes centros educativos.

Figura 2. Esquema de funcionamiento del sistema de telemetría

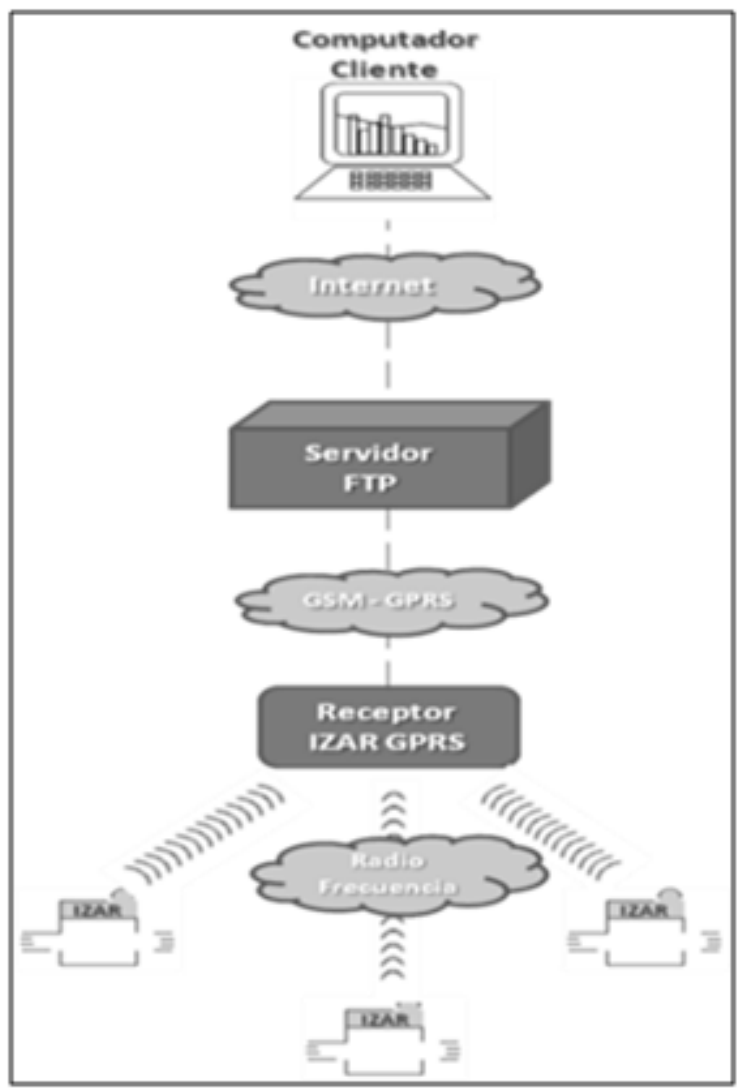

Fuente: los autores. 2011.

\section{RESULTADOS Y DISCUSIÓN}

La red primaria de suministro de agua potable está compuesta en su mayoría por tubería galvanizada en diámetro de dos pulgadas (2"), que abastece la red secundaria compuesta por tubería PVC de tres cuartos de pulgada $(3 / 4)$. El sistema de abastecimiento funciona por bombeo, el cual impulsa el agua hasta la azotea del tercer nivel, donde se encuentran ubicados cuatro tanques de almacenamiento con capacidad de quinientos (500) litros cada uno. El agua es distribuida a las unidades hidrosanitarias por gravedad (Figura 3). 


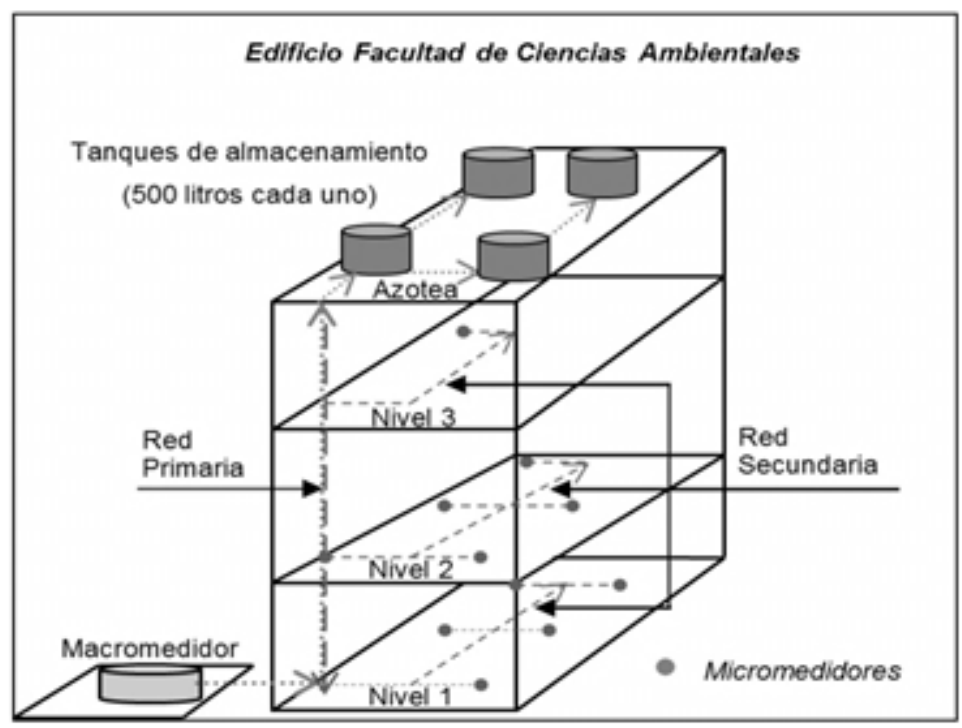

Figura 3. Red de distribución de agua Facultad de Ciencias Ambientales.

Fuente: los autores. 2012.

La FCA cuenta con 97 instalaciones hidrosanitarias, de las cuales el $71 \%$ corresponde a grifos, el $16 \%$ a inodoros, el $7 \%$ a duchas y el $6 \%$ a urinarios (Tabla 1 ).

Tabla 1. Instalaciones hidrosanitarias FCA

\begin{tabular}{|c|c|c|c|c|c|c|}
\hline \multirow{2}{*}{ Instalaciones } & Grifos & \multirow{2}{*}{\begin{tabular}{|l|} 
Urinarios \\
Hombres
\end{tabular}} & \multicolumn{2}{|c|}{ Inodoros } & \multicolumn{2}{|c|}{ Duchas } \\
\hline & Hombres Mujeres & & Hombres & Mujeres & Hombres & Mujeres \\
\hline Baños & 6 & 6 & 7 & 8 & 1 & 1 \\
\hline Pocetas & 3 & 0 & 0 & 0 & & 0 \\
\hline Laboratorios & 52 & 0 & 0 & 0 & & 5 \\
\hline Cocineta & 1 & 0 & & 0 & & 0 \\
\hline Jardín & 1 & 0 & & 0 & & 0 \\
\hline Total & 69 & 6 & & 15 & & 7 \\
\hline
\end{tabular}

De acuerdo con la inspección física realizada durante el periodo de estudio, el $90 \%$ de las instalaciones hidrosanitarias no presentaron fugas, el $7 \%$ registró fugas importantes y el $3 \%$ registró goteos, estas unidades requieren mantenimiento o reparación.

\section{Usos del agua}

Los usos de agua identificados en la FCA son: aseo personal de estudiantes, docentes y administrativos, limpieza y aseo del edificio, agua para consumo, agua para laboratorios y riego de jardines. En la figura 4 se ilustra el perfil del consumo de agua en la FCA, donde se aprecian las pérdidas físicas entre las 23:00 y las 5:00 horas del día siguiente, tiempo en el cual no existe ocupación en las instalaciones del centro educativo. Este perfil de consumo corresponde a las mediciones realizadas en todos los puntos de consumo del edificio. 
Figura 4. Perfil del consumo de agua en la Facultad de Ciencias Ambientales.

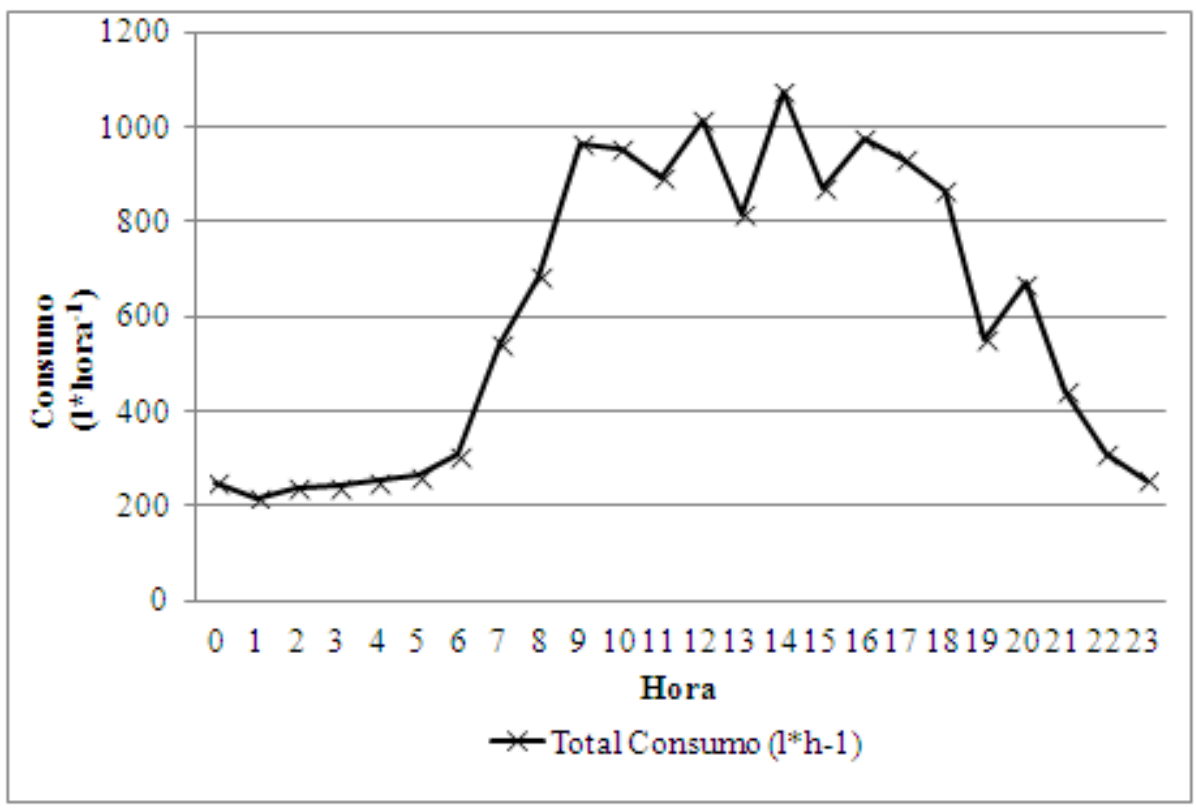

Fuente: los autores. 2014.

En la Tabla 2 se muestran los promedios de consumo bruto y neto para cada uso en el periodo de observación, con estos valores se calculan los indicadores de referencia (bruto y neto).

Estos indicadores se generan a partir de las diferentes formas en que se expresa el consumo de agua por unidad evaluada. Así, la dotación bruta corresponde a la cantidad de agua requerida para satisfacer las necesidades básicas de un usuario, considerando las pérdidas que ocurran en el sistema de distribución de agua; ahora bien, cuando no se consideran las pérdidas del sistema se obtiene la dotación neta (Ministerio de Desarrollo Económico, 2000). La estimación de las pérdidas para cada unidad hidrosanitaria, se calculó determinando los consumos de agua entre las 23:00 y las 5:00 horas del día siguiente, teniendo en cuenta que en este horario no existen actividades que demanden agua en la institución. 
Tabla 2. Descripción de los usos de agua en la FCA

\begin{tabular}{|c|c|c|c|c|c|}
\hline \multicolumn{2}{|c|}{ Uso de agua } & $\begin{array}{c}\text { Consumo } \\
\text { bruto } \\
\left(l^{*} d^{-1}\right)\end{array}$ & $\begin{array}{c}\text { Indicador } \\
\text { bruto }\end{array}$ & $\begin{array}{l}\text { Consumo } \\
\text { neto } \\
\left(I^{*} d^{-1}\right)\end{array}$ & $\begin{array}{l}\text { Indicador } \\
\text { neto }\end{array}$ \\
\hline \multirow{5}{*}{$\begin{array}{c}\text { Aseo } \\
\text { personal }\end{array}$} & \multirow{3}{*}{ estudiantes } & \multirow{3}{*}{11476} & 11,5 & \multirow{3}{*}{10444} & 10,5 \\
\hline & & & $\left(l^{*}\right.$ pers $s^{-1 *} d t$ & & $\left(I^{*}\right.$ pers $s^{-1 *} d t$ \\
\hline & & & 1) & & 1) \\
\hline & \multirow[b]{2}{*}{$\begin{array}{c}\text { docentes y } \\
\text { administrativos }\end{array}$} & \multirow[b]{2}{*}{1066} & 31,3 & \multirow[b]{2}{*}{1055} & 31,0 \\
\hline & & & $\begin{array}{c}\left(l^{*} \text { pers } s^{-1 *} d t\right. \\
1)\end{array}$ & & $\begin{array}{c}\left(l^{*} \text { pers }{ }^{-1 *} d t\right. \\
\text { 1) }\end{array}$ \\
\hline \multirow{5}{*}{$\begin{array}{l}\text { Preparación } \\
\text { de } \\
\text { alimentos }\end{array}$} & cafetería & \multirow{3}{*}{178} & 5,2 & \multirow{3}{*}{178} & 5,2 \\
\hline & docentes y & & $\left(l^{*}\right.$ pers $s^{-1 *} d t$ & & $\left(l^{*}\right.$ pers $s^{-1 *} d t$ \\
\hline & administrativos & & 1) & & 1) \\
\hline & \multirow{2}{*}{$\begin{array}{c}\text { Dispensador } \\
\text { de agua }\end{array}$} & \multirow[b]{2}{*}{200} & 0,20 & \multirow[b]{2}{*}{188} & 0,19 \\
\hline & & & $\begin{array}{l}\left(1 * p^{*} e^{-1 *} d t\right. \\
\text { 1) }\end{array}$ & & $\begin{array}{l}\left(l^{*} \text { pers }{ }^{-1 *} d t\right. \\
\text { 1) }\end{array}$ \\
\hline \multirow{2}{*}{\multicolumn{2}{|c|}{ Limpieza }} & \multirow{2}{*}{250} & 0,05 & \multirow{2}{*}{250} & 0,05 \\
\hline & & & $\left(I^{*} m^{-2 *} d^{-1}\right)$ & & $\left(l^{*} m^{-2 *} d^{-1}\right)$ \\
\hline \multicolumn{2}{|c|}{ Riego para jardín (*) } & 450 & $\begin{array}{c}45 \\
\left(x^{*} m^{-2 *} c^{-1}\right)\end{array}$ & 450 & $\begin{array}{c}45 \\
\left(*^{*} m^{-2 *} c^{-1}\right)\end{array}$ \\
\hline \multirow{3}{*}{\multicolumn{2}{|c|}{ Laboratorios $\left(^{\star \star}\right)$}} & \multirow{3}{*}{936} & 9,4 & & 9,3 \\
\hline & & & $\left(l^{*}\right.$ muestra- & 931 & $\left(I^{\star}\right.$ muestra* \\
\hline & & & 1) & & 1) \\
\hline \multirow{2}{*}{$\begin{array}{c}\text { Pérdidas de } \\
\text { agua }\end{array}$} & Físicas & 1060 & - & - & \multirow{2}{*}{$13,4 \%$} \\
\hline & No registradas & 1028 & - & - & \\
\hline \multicolumn{2}{|c|}{ Totalizador } & 15584 & - & - & - \\
\hline \multicolumn{2}{|c|}{ Demanda Total } & 15584 & - & 13495 & - \\
\hline
\end{tabular}

La Norma Técnica Colombiana NTC 1500:2004 establece valores de 50 litros usuario${ }^{1 *}$ día $^{-1}$ para instituciones educativas. Sin embargo, estos consumos dependen de las situaciones particulares de las instituciones. Algunos valores reportados muestran dotaciones de 21,4; 41 y 35 litros $^{*}$ usuario $^{-1 *}$ día $^{-1}$ en las universidades de los Andes, Antioquia y Valle del Cauca, respectivamente. Para el caso del presente estudio, el consumo bruto es de 15,1 litros $^{*}$ usuario $^{-{ }^{*}}$ día $^{-1}$, que equivale al $12 \%$ del consumo doméstico para Pereira (Morales-Pinzón, 2012b), mientras el consumo neto es de 13,1 litros ${ }^{*}$ usuario $^{-{ }^{*}} \mathrm{dia}^{-1}$. La variación en el valor del indicador se debe, en primera medida, a los usos de agua en cada institución, que corresponden con las actividades que se realizan diariamente cumpliendo su objeto misional.

De acuerdo con la Tabla 2, a la FCA ingresan en promedio 15.584 litros $^{*}$ día $^{-1}$, los cuales son demandados según sus usos, como se muestra en la figura 5 . 
Figura 5. Demanda de agua por usos en la FCA.

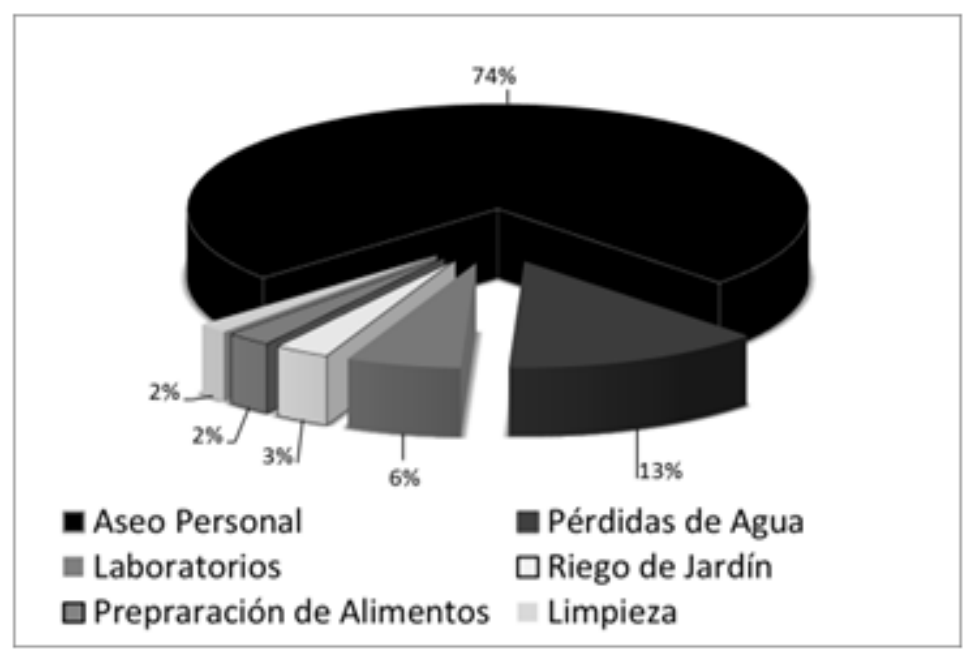

Fuente: Ios autores. 2012.

\section{Modelo propuesto de cálculo de la demanda}

A partir de los datos se encontraron expresiones que pueden ser aplicadas al cálculo de la demanda de agua en edificios educativos. En las ecuaciones 1,2 y 3 se presentan los resultados de los modelos propuestos para el cálculo de la demanda de agua en centros educativos, en función de los usos que en ella se presentan (Tabla 3). Esta propuesta de modelo matemático de consumo debe ser validada con base en las experiencias de otras instituciones para así definir y ajustar su incertidumbre. En principio, el mejor modelo está representado por la ecuación 2 con un coeficiente de determinación $\left(R^{2}\right)$ de 0,95 y un porcentaje de error medio cuadrático (EMC) de $10 \%$. Este resultado evidencia que la variable clave para la estimación de la demanda de agua en edificaciones educativas es el número de estudiantes.

Tabla 3. Modelos matemáticos para la estimación de la demanda neta en instituciones educativas

\begin{tabular}{cccc}
\hline & Modelo & $\mathrm{R}^{2}$ & \%EMC \\
\hline Eq. 1 & $D c e=365,5+10,7 \times E+42,5 \times D+0,1 \times L+52,1 \times R+10,2 \times M$ & 0,90 & $20 \%$ \\
Eq. 2 & $D c e=(10,7 \times E+42,1 \times D+0,1 \times L+51,6 \times R+10 \times M) /(1-P)$ & 0,95 & $10 \%$ \\
Eq. 3 & $D c e=2187,5+14,9 \times E$ & 0,92 & $17 \%$ \\
\hline
\end{tabular}

Donde:

$\begin{array}{cl}E & \text { Número de estudiantes (Est) } \\ D & \text { Número de docentes (Doc) } \\ L & \text { Área de limpieza del centro educativo }\left(m^{2}\right) \\ R & \text { Área de riego para jardín y zonas verdes }\left(m^{2}\right) \\ M & \text { Números de muestras procesadas en el laboratorio (\#muestras) } \\ P & \text { Pérdidas de agua (\%) } \\ D c e & \text { Demanda de agua en centros educativos }\left(l^{*} c^{-1}\right)\end{array}$


Con el fin de validar los modelos matemáticos propuestos, con la información de la FCA, en la tabla 4 se presentan las variables utilizadas en cada uno de ellos y los resultados de la demanda neta de la institución educativa.

Tabla 4. Estimación de la demanda neta para la FCA usando modelos propuestos.

\begin{tabular}{|c|c|c|c|c|c|c|c|}
\hline \multirow[b]{2}{*}{$\begin{array}{l}\text { Varia } \\
\text { ble }\end{array}$} & \multirow[b]{2}{*}{ Descripción } & \multirow[b]{2}{*}{$\begin{array}{c}\text { Unidad } \\
\text { es }\end{array}$} & \multirow[b]{2}{*}{ Valor } & \multicolumn{4}{|c|}{$\begin{array}{c}\text { Demanda de agua neta en } \\
\text { centros educativos (itros }{ }^{*} \text { di }^{-1} \text { ) }\end{array}$} \\
\hline & & & & $\begin{array}{c}\text { Model } \\
01\end{array}$ & $\begin{array}{c}\text { Model } \\
02\end{array}$ & $\begin{array}{c}\text { Modelo } \\
3\end{array}$ & Real \\
\hline$E$ & $\begin{array}{l}\text { Número de } \\
\text { estudiantes }\end{array}$ & $\begin{array}{l}\text { Person } \\
\text { as } \\
\text { Person }\end{array}$ & 997 & & & & \\
\hline$D$ & $\begin{array}{l}\text { Número de docentes } \\
\text { Area de limpieza del }\end{array}$ & $\begin{array}{l}\text { as } \\
m^{2}\end{array}$ & 34 & & & & \\
\hline$L$ & $\begin{array}{l}\text { centro educativo } \\
\text { Area de riego para } \\
\text { jardín y zonas verdes }\end{array}$ & $m^{2}$ & 10 & 14.485 & 16.284 & 17.012 & $\begin{array}{c}15.5 \\
84\end{array}$ \\
\hline$M$ & $\begin{array}{l}\text { Número de muestras } \\
\text { procesadas en e } \\
\text { laboratorio }\end{array}$ & $\begin{array}{l}\text { \#muestr } \\
\text { as }\end{array}$ & 100 & & & & \\
\hline$P$ & Pérdidas de agua & $\%$ & $\begin{array}{r}13,4 \\
\%\end{array}$ & & & & \\
\hline Diferer & ncia con valor real & $\%$ & & $-7,1 \%$ & $4,5 \%$ & $9,2 \%$ & \\
\hline
\end{tabular}

Fuente: Ios autores. 2015

\section{CONCLUSIONES}

Los criterios de referencia de consumo de agua para centros educativos establecidos en la normatividad colombiana (NTC 1500 de 2004) no reflejan la realidad, regulando valores superiores a los consumos actuales de estas instituciones. Dicha condición puede llevar por una parte al sobre dimensionamiento de las estructuras de suministro de agua y por otra a una señal equívoca para programas de uso eficiente de agua.

Este trabajo es un aporte inicial en la determinación de patrones de consumo en centros educativos, que se debe validar en otras instituciones para estandarizar umbrales de consumo de agua de acuerdo con sus usos en instituciones de educación.

Para la -FCA, el presente trabajo se convierte en una herramienta de gestión, que permitirá evaluar la tendencia del uso del agua en la institución y verificar el desempeño de los programas implementados con el fin de optimizar el uso del recurso, que indiscutiblemente conlleva beneficios económicos y ambientales. 
Los resultados encontrados en el presente estudio son el punto de partida para la búsqueda de criterios de desmpeño de uso de agua en instituciones educactivas que se pueden determinar realizando optimizaciones, tecnológicas o culturales, en las diferentes formas de aprovechamiento del recurso.

\section{REFERENCIAS}

- Bonnet, J., Devel, C., Faucher, P., y Roturier, J. (2002). Analysis of electricity and water end-uses in university campuses: case-study of the University of Bordeaux in the framework of the ecocampus European collaboration. Journal of Cleaner Production, 10, pp. 13-24.

- Cheng, C. y Hong, Y. (2004). Evaluating water utilization in primary schools.Building and Environment, 39, pp. 837-845.

- Manco-Silva, D., Guerrero, J., Ocampo, A. (2012). Eficiencia en el uso del agua residencial. Revista Ingenierías Universidad de Medellín, 11, pp. 23-38.

- Jorgensen, B., Graymore, M., y O'toole, K. (2009). Household water use behavior: An integrated model. Journal of Environmental Management, 91, pp. 227-236.

- Ministerio de Desarrollo Económico. (2000). Reglamento Técnico para el sector Agua Potable y Saneamiento Básico(RAS). En: Resolución 1096 de 2000. Colombia.

- Morales-Pinzón, T., Rieradevall, J., Gasol M., Gabarrell, C. (2012a). Plugrisost v1.0: Modelo dinámica de flujos de agua para uso doméstico, con énfasis en aprovechamiento de fuentes no convencionales (pluviales, grises). Recuperado de: http://sostenipra.ecotech.cat/login.php?referrer=plugrisost . (2012b). Potential of rainwater resources based on urban and social aspects in Colombia. Water Environment Journal, 26, pp. 550-559.

- Trujillo, C., y Sarmiento, J. (2012). Estrategias de uso eficiente y ahorro de agua en centros educativos, caso de estudio, edificio Facultad de Ciencias Ambientales. Universidad Tecnológica de Pereira. 


\section{AGRADECIMIENTOS}

Los autores desean expresar sus agradecimientos a los proyectos "Diseño y evaluación de estrategias de uso eficiente y ahorro de agua en centros educativos caso de estudio campus de la Universidad Tecnológica de Pereira" desarrollado por el grupo de investigación en Agua y Saneamiento -GIAS y "Metabolismo Urbano y Análisis Ambiental del aprovechamiento de Agua no Convencional en Edificaciones más Sostenibles" desarrollado por el grupo de investigación en Gestión Ambiental Territorial -GAT, financiados por la Vicerrectoría de Investigaciones, Innovación y Extensión, Universidad Tecnológica de Pereira, Colombia, mediante convocatoria interna de proyectos de los años 2012 y 2013.

\section{POTENCIAL CONFLICTO DE INTERÉS}

No hay ningún tipo de conflicto de interés por publicación de este artículo, el cual se elaboró mediante proyectos desarrollados por los Grupos de Investigación en Agua y Saneamiento y en Gestión Ambiental Territorial, de los cuales hacen parte los autores.

\section{FUENTES DE FINANCIACIÓN}

Se agradece a la Universidad Tecnológica de Pereira y su Vicerrectoría de Investigaciones, Innovación y Extensión por la financiación mediante convocatoria interna de los proyectos que permitieron recolectar la información para la elaboración del actual artículo de investigación.

1. Administrador Ambiental, Universidad Tecnológica de Pereira, Pereira, Colombia. Magíster en Ecotecnología, Universidad Tecnológica de Pereira, Pereira, Colombia. Docente. Grupo de Investigación en Agua y Saneamiento -GIAS. Universidad Tecnológica de Pereira, Pereira, Colombia.davis@utp.edu.co. http://orcid.org/00000003-3841-0067

2. Ingeniero Sanitario, Universidad del Valle, Santiago de Cali, Colombia. Magíster en Ingeniería Sanitaria y Ambiental, Universidad del Valle, Santiago de Cali, Colombia. Doctor en Ingeniería, Technische Universitat Berlin, Berlín, Alemania. Docente Titular. Grupo de Investigación en Agua y Saneamiento -GIAS. Universidad Tecnológica de Pereira, Pereira, Colombia. jhguerre@utp.edu.co. http://orcid.org/0000-0002-02432320

3. Administrador Ambiental, Universidad Tecnológica de Pereira, Pereira, Colombia. Magíster en Investigación Operativa y Estadística, Universidad Tecnológica de Pereira, Pereira, Colombia. Doctor en Ciencia y Tecnología Ambientales, Universidad Autónoma de Barcelona, Barcelona, España. Docente Asociado. Grupo de Investigación en Gestión Ambiental Territorial -GAT. Pereira, Colombia.tito@utp.edu.co. http://orcid.org/0000-0003-3156-225 
Para citar este artículo: Manco-Silva, D.; Guerrero-Erazo, J. y Morales-Pinzón, T. (2017). Estimación de la demanda de agua en centros educativos: caso de estudio Facultad de Ciencias Ambientales de la Universidad Tecnológica de Pereira, Colombia. Luna Azul, 44, 153-164. DOI: 10.17151/luaz.2017.44.9. Recuperado de http://200.21.104.25/lunazul/index.php/component/content/article?id=225

Esta obra está bajo una Licencia de Creative Commons Reconocimiento CC BY

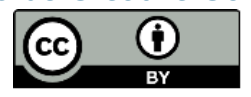

